\title{
RAC1-GTP promotes epithelial-mesenchymal transition and invasion of colorectal cancer by activation of STAT3
}

\author{
Kai Zhou ${ }^{1,2} \cdot$ Jun Rao $\mathbb{1}^{1,2,3} \cdot$ Zhi-hua Zhou ${ }^{1,2} \cdot$ Xiao-hong Yao, ${ }^{1,2}$ Feng $\mathrm{Wu}^{1,2} \cdot$ Jing Yang ${ }^{1,2} \cdot$ Lang Yang ${ }^{1,4}$. \\ Xia Zhang ${ }^{1,2} \cdot$ You-hong Cui ${ }^{1,2} \cdot$ Xiu-Wu Bian $\mathbb{1}^{1,2} \cdot$ Yu Shi ${ }^{1,2} \cdot$ Yi-fang Ping ${ }^{1,2}$
}

Received: 6 August 2017 / Revised: 4 April 2018 / Accepted: 5 April 2018 / Published online: 8 June 2018

(c) United States \& Canadian Academy of Pathology 2018

\begin{abstract}
Epithelial-mesenchymal transition (EMT) plays a critical role in initiating tumor invasion and metastasis of colorectal cancer (CRC), although the underlying mechanisms remain to be clarified. Herein, we demonstrate that the active form of Rac family small GTPase 1 (RAC1-GTP) is overexpressed in CRCs and promotes the EMT-mediated invasion of CRC cells through activation of the signal transducers and activators of transcription 3 (STAT3) pathway. Increased expression of RAC1-GTP in CRC tissues was positively correlated with the TNM stages of CRCs and indicated poor prognosis of CRC patients. Targeting RAC1-GTP activity by its specific inhibitor NSC23766 markedly suppressed the migration and invasion of CRC cells. Mechanistically, RAC1-GTP directly interacted with STAT3 to promote STAT3 phosphorylation, thus promoted EMT of CRC cells. Enforced expression of constitutively activated STAT3 (STAT3-C) abrogated the suppressive effect of RAC1-GTP disruption on the migration and invasion of CRC cells. Importantly, NSC23766 disrupted EMT in CRC cells and significantly diminished growth of CRC xenografts. Taken together, our data indicate that RAC1-GTP is an important player in EMT-mediated tumor invasion and a potential therapeutic target for CRCs.
\end{abstract}

Electronic supplementary material The online version of this article (https://doi.org/10.1038/s41374-018-0071-2) contains supplementary material, which is available to authorized users.

$凶$ Xiu-Wu Bian
bianxiuwu@263.net
$\square$ Yu Shi
drshiyu@126.com
$\square$ Yi-fang Ping
pingyifang@126.com

1 Institute of Pathology and Southwest Cancer Center, Southwest Hospital, Third Military Medical University (Army Medical University), Chongqing 400038, China

2 Key Laboratory of Tumor Immunopathology, Ministry of Education of China, Chongqing 400038, China

3 Department of Hematology, Xinqiao Hospital, Third Military Medical University (Army Medical University), Chongqing 400038, China

4 Department of Gastroenterology, Army General Hospital of PLA, Beijing 100700, China

\section{Introduction}

Colorectal cancer (CRC) is the fourth most common cause of cancer-related death worldwide [1]. Invasion and metastasis are the major contributors for the poor outcome of patients with CRC [2]. Although the 5-year survival rate of patients diagnosed with localized CRC is $90.1 \%$, it drops to $11.7 \%$ when tumors spread to distant organs [3]. Therefore, understanding the mechanisms underlying CRC invasion and metastasis is critical for developing novel agents and therapeutic strategies to improve the clinical outcome of CRC patients.

Epithelial-mesenchymal transition (EMT) plays a critical role in promoting tumor invasion and metastasis in CRC [4]. During the EMT process, the epithelial marker Ecadherin is downregulated and the mesenchymal marker $\mathrm{N}$ cadherin is upregulated. This process, referred to as "cadherin switching", destroy intercellular adhesion and enables cells to acquire invasive and migratory capacities [5]. It has been reported that several signal pathways, such as JAK/ STAT, Wnt, and PI3K/AKT, are involved in the regulation of the EMT process in CRC [6-8], but the molecular mechanism underlying the initiation of EMT in CRC needs to be further explored. 
Rho GTPases are small GTP-binding proteins that actively participate in the regulation of cytoskeletal dynamics and activation of protein kinases, which are crucial for the initiation of EMT. As a well-characterized member of Rho GTPases family, RAC1 has been shown to regulate diverse cellular events such as membrane ruffling, mitogenesis, cytoskeletal rearrangement, and migration [9-11]. RAC1 has two conformations, active (RAC1-GTP) and inactive (RAC1-GDP) [12]. The transition from RAC1-GDP to RAC1-GTP, which is regulated by guanine nucleotide exchange factors, leads to the activation of several downstream signaling pathways. For example, RAC1-GTP can activate signal transducers and activators of transcription 3 (STAT3) by activating the IL-6/JAK pathway [13] or by directly binding to STAT3 [14]. It has also been reported that RAC1-GTP can activate the MAPK pathway in breast cancer [15]. However, the role of RAC1-GTP in EMT of $\mathrm{CRC}$ and its clinical relevance remain largely unknown.

In this study, we demonstrate that inhibition of RAC1-GTP disrupted the EMT process and suppressed CRC migration and invasion by attenuating the phosphorylation of STAT3. RAC1-GTP expression was positively correlated with the tumor/node/metastasis (TNM) stage of CRC and predicted poor prognosis for CRC patients. Our study underscores RAC1-GTP as a new regulator of EMT, with potential prognostic and therapeutic significance for patients with CRC.

\section{Materials and methods}

\section{Cell culture}

Human CRC cell lines HCT116, SW480, and SW620 from American Type Culture Collection were authenticated by short tandem repeat profiling (GenePrint 10 System, B9510, Promega, WI) and were passaged for $<6$ months. Cells were maintained as monolayer culture in Dulbecco's modified Eagle medium (DMEM; Gibco, Grand island, NY) containing $10 \%$ fetal bovine serum (FBS) and were incubated at $37^{\circ} \mathrm{C}$ in a humidified incubator with $5 \% \mathrm{CO}_{2}$.

\section{Patients and tissue specimens}

A total of 147 formalin-fixed and paraffin-embedded (FFPE) surgical cancerous tissues and the corresponding adjacent normal tissues were collected from CRC patients from Southwest Hospital from 2002 to 2008. All patients had not received radiotherapy, chemotherapy, or immunotherapy before surgery. All the specimens were diagnosed by at least two senior pathologists (YFP and JY) according to the World Health Organization classification. Follow-up information was available for a period of a minimum period of 80 months. The study was approved by the Southwest
Hospital Research Ethics Committees, and all patients were enrolled by written informed consent. A patient cohort containing $380 \mathrm{CRC}$ cases and 50 adjacent normal cases from The Cancer Genome Atlas (TCGA) database (https:// tcga-data.nci.nih.gov/tcga) was applied to analyze the gene expression of RAC1. This study was conducted according to the principles of the Helsinki Declaration.

\section{Lentiviral infection}

The lentiviral construct expressing Flag-tagged constitutively activated STAT3 (STAT3-C) was constructed by cloning STAT3-Flag into a pCDH-MCS-T2a-Puro-MSCV vector (System Bioscience). Lentivirus packaging and transduction were performed as previously described [16]. The CRC cells stably expressing STAT3-C were enriched by puromycin selection for positive clones.

\section{Cell proliferation assay}

Cell proliferation was measured using Cell Counting Kit-8 (CCK-8; Beyotime, Shanghai, China). Briefly, tumor cells (1000 cells/well) were seeded into 96-well plates and cultured in medium with or without NSC23766 $(100 \mu \mathrm{M})$ at $37^{\circ} \mathrm{C}$ for 1 week. The culture medium was removed and $100 \mu \mathrm{L}$ fresh DMEM containing $10 \mu \mathrm{L}$ of CCK-8 solution was added at intervals. After incubation for $1 \mathrm{~h}$, the absorbance at $450 \mathrm{~nm}$ was measured with a microtiter plate reader (Thermo Fisher Scientific) and the results were expressed as optical density.

\section{Quantitative real-time PCR}

Quantitative real-time PCR was performed as previously described [17]. The sequences of the specific primer sets for Snail, Slug, E-cadherin, N-cadherin, MMP9, Vimentin, and $\beta$-actin are listed in Supplementary Table 1 . The level of $\beta$ actin mRNA was used as the internal control.

\section{Western blot}

Western blot analysis was conducted as previously described [18]. Primary antibodies are listed in Supplementary Table 2. Secondary antibodies were horseradish peroxidase (HRP)-labeled goat anti-rabbit $\operatorname{IgG}(\mathrm{H}+\mathrm{L})$ (A0208, 1:5000, Beyotime), HRP-labeled goat anti-mouse $\operatorname{IgG}$ $(\mathrm{H}+\mathrm{L})(\mathrm{A} 0216,1: 5000$, Beyotime), and goat anti-mouse IgM (BA1075, BOSTER, 1:5000, Wuhan, China).

\section{Co-immunoprecipitation}

Co-immunoprecipitation (Co-IP) was performed using the Thermo Scientific Pierce Co-IP kit (26149) following the manufacturer's protocol. Briefly, mouse anti-human active 
RAC1 monoclonal antibody (mAb) (26903, NewEast Biosciences), mouse anti-human STAT3 mAb (\#9139, Cell Signaling Technology), or an IgG control antibody (\#3900, Cell Signaling Technology) was immobilized for $2 \mathrm{~h}$ using AminoLink Plus coupling resin. The resin was then incubated with cells lysate overnight and proteins were eluted using elution buffer. Samples were analyzed by western blot using anti-human active RAC1 mAb (26903, 1:800, NewEast Biosciences) and mouse anti-human STAT3 monoclonal antibody (\#9139, Cell Signaling Technology).

\section{Detection of active RAC1}

The activation of RAC1 was assessed by Active RAC1 Detection Kit (\#8815, Cell Signaling Technology) according to the manufacturer's instruction. Briefly, cells were washed with ice-cold phosphate-buffered saline and lysed with lysis buffer that was freshly added with $1 \mathrm{mM}$ phenylmethanesulfonyl fluoride (\#8553, Cell Signaling Technology) on ice for $5 \mathrm{~min}$. After centrifugation at $16000 \times g$ for $15 \mathrm{~min}$ at $4{ }^{\circ} \mathrm{C}$, the supernatant was mixed with GSTPAK1-PBD/glutathione resins and incubated at $4{ }^{\circ} \mathrm{C}$ for $1 \mathrm{~h}$. Then the resin was washed three times with $1 \times$ cell lysis/ binding/wash buffer. The resin-bound GTP-RAC1 was eluted with $2 \times$ SDS sample buffer containing $200 \mathrm{mM}$ dithiothreitol followed by western blot analysis with a mouse against human RAC1 mAb (\#8631, 1:1000, Cell Signaling Technology). Total RAC1 in cell lysate was also determined by western blotting.

\section{Cell migration and invasion assay}

Transwell chambers with $8 \mu \mathrm{m}$ pore size were used to determine the migratory and invasive capabilities of the CRC cells. Chambers pre-coated with Matrigel (\#356234, Corning, Corning, NY) were used for evaluation of cell invasion. CRC cells treated with or without NSC23766 $(100 \mu \mathrm{M})$ or BP-1-102 $(20 \mu \mathrm{M})$ were seeded in the upper chamber. The lower chamber contained DMEM with $10 \%$ FBS. After $12 \mathrm{~h}$ of incubation, non-migratory cells in the upper chambers were wiped off and the chambers were fixed with $4 \%$ paraformaldehyde and stained with a Crystal Violet Staining Solution (C0121, Beyotime). Migrating or invading cells in the lower chamber were counted in five randomly selected fields under a light microscope at $\times 400$ magnification.

\section{Xenograft experiments}

Six-week-old male nude mice were subcutaneously injected with $1 \times 10^{6}$ HCT116 CRC cells in each left and right lateral flank. Six days after injection, mice were treated with NSC23766 (2.5 mg/kg, intraperitoneal every day) or vehicle control (dimethylsulfoxide) [19]. Three weeks later, tumors were harvested and weighed. Tumor volume was calculated using the following formula: volume $=$ length $\times$ width $^{2} \times 1 / 2$. The animal experiments were approved by the Institutional Animal Care and Use Committee of Southwest Hospital, Third Military Medical University in accordance with the Guide for the Care and Use of Laboratory Animals of this institution.

\section{Immunohistochemistry}

Immunohistochemical staining was performed using DAKO REAL $^{\mathrm{TM}}$ EnVision $^{\mathrm{TM}}$ Detection System (DAKO, Santa Clara, CA) as previously described [20]. Briefly, the FFPE specimens were cut into $4 \mu \mathrm{m}$-thick sections. After dewaxing, hydration, heat-induced antigen retrieval, and blocking of endogenous peroxidase activity, the sections were incubated with primary antibodies, including mouse anti-human active RAC1 (26903, 1:600, NewEast Biosciences), mouse anti-human E-cadherin (MAB-0589, Maxim, Fuzhou, China), rabbit anti-human Slug (s-1382R, Bioss, Beijing, China), and rabbit anti-human phospho-STAT3 (Tyr705) (\#9145, Cell Signaling Technology) at $4{ }^{\circ} \mathrm{C}$ overnight. The sections were then incubated with secondary antibody (DK2600, Glostrup, Denmark) at $37^{\circ} \mathrm{C}$ for $30 \mathrm{~min}$, and visualized using diaminobenzidine solution (DAKO) and lightly counterstained with hematoxylin. Immunohistochemical scores of RAC1-GTP, E-cadherin, and Slug were independently semi-quantified by two pathologists (YFP and JY) according to the staining intensity and percentage of positive tumor cells as previously described [21].

\section{Statistical analysis}

All statistical analyses were performed using SPSS 18.0 software. The unpaired Student's $t$-test was used to determine the statistical difference between two groups. The correlation between RAC1-GTP expression and pathological features of patients were analyzed by Pearson's $\chi^{2}$ test. The Kaplan-Meier method with log-rank test was used for patient survival analysis. Cox's proportional hazard model was used for univariate and multivariate survival analysis. Data were presented as the mean $\pm \mathrm{SD}$. Statistical significance was set at $p<0.05$.

\section{Results}

\section{Overexpression of RAC1-GTP is correlated with TNM stage in CRCs and predicts poor prognosis of CRC patients}

To determine the pathological significance of RAC1-GTP in CRC, we examined RAC1-GTP expression in CRC 

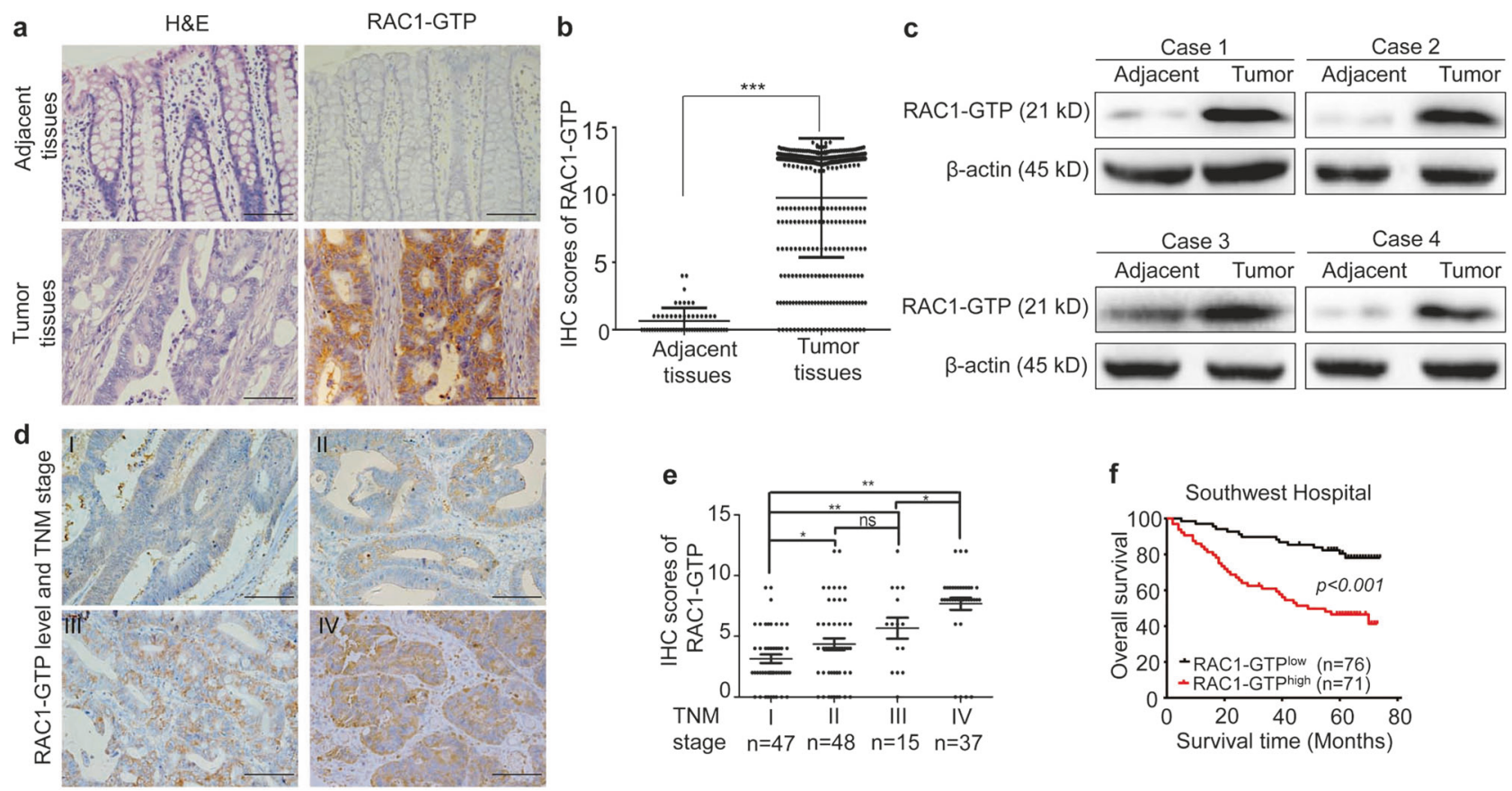

Fig. 1 Overexpression of RAC1-GTP is correlated with TNM stages of CRCs and predicts poor prognosis of CRC patients. a Representative images of H\&E staining and IHC staining of RAC1-GTP in CRC tumor tissues and adjacent normal tissues. Scale bar $=100 \mu \mathrm{m}$. b $H$-scored quantification of IHC staining for RAC1-GTP. c Western blot analysis of RAC1-GTP in tumor and adjacent normal tissues. d

cancerous tissues and adjacent normal tissues by immunohistochemical (IHC) staining with an antibody that specifically labels RAC1-GTP (Figure S1a and b). As shown in Fig. 1a, RAC1-GTP was preferentially expressed in CRC cancerous tissues but not in the adjacent normal tissues. Accordingly, the IHC scores of RAC1-GTP were significantly higher in cancer tissues than in adjacent normal tissues $(p<0.001$; Fig. 1b). The differential expression of RAC1-GTP was confirmed by western blot analyses in four fresh CRC specimens (Fig. 1c).

We next analyzed the association of RAC1-GTP expression and clinicopathological parameters of CRC patients. As shown in Fig. 1d, e, the expression level of RAC1-GTP in cancerous tissues was increased with TNM stages (stage II vs. stage I, $p<0.05$; stage III vs. stage I, $p<$ 0.01 ; stage IV vs. stage I, $p<0.01$; stage III vs. stage IV, $p$ $<0.05$, respectively). No statistical difference in RAC1GTP expression was observed between stage II and stage III CRCs $(p=0.063$, Fig. 1e), which might attribute to a limited number of stage III cases in the cohort. Also, RAC1GTP expression was positively correlated with the depth of tumor invasion, distant metastasis, lymph node metastasis, and tumor recurrence status (Table 1).

Kaplan-Meier analysis showed that patients with high RAC1-GTP expression exhibited shorter overall survival compared to those with low RAC1-GTP expression $(p<$
Representative IHC staining of RAC1-GTP in CRC samples at different TNM stages. Scale bar $=100 \mu \mathrm{m}$. e IHC scores of RAC1-GTP expression in the tumors of all stages. $\mathbf{f}$ Kaplan-Meier analysis of the correlation between RAC1-GTP and overall survival in 147 CRC patients. $* p<0.05 ; * * p<0.01 ; * * * p<0.001$; ns not significant

0.001; Fig. 1f). Univariate and multivariate Cox analyses showed that RAC1-GTP expression was an independent prognostic indicator for CRC patients (Table 2). Notably, we compared the expression of RAC1 mRNA in nine fresh CRC specimens, and found that there was no significant difference between the cancerous and adjacent normal tissues (Figure S1c). Analysis of CRC samples from TCGA database also showed similar results (Figure S1d), implying that RAC1GTP but not RAC1 is involved in CRC progression. These results suggest that RAC1-GTP may be an important player in CRC progression and may have prognostic significance.

\section{RAC1-GTP fosters the migration and invasion of CRC cells in vitro}

Since RAC1-GTP was associated with the invasion and metastasis in CRC specimens, we further investigated the effect of RAC1-GTP on the migration and invasion of CRC cells in vitro. Treatment with NSC23766, a specific inhibitor for RAC1-GTP, markedly disrupted RAC1-GTP expression in HCT116, SW480, and SW620 cells (Fig. 2a and S2a). Additionally, NSC23766 treatment significantly attenuated the migration and invasion capabilities of the cells compared to treatment with the vehicle. The migrating cells were $90 \pm 47$ vs. $179 \pm 24$ for HCT116, $111 \pm 8$ vs. $258 \pm 29$ for SW480, and $170 \pm 14$ vs. $284 \pm 31$ for SW620 
Table 1 Association of RAC1-GTP expression and clinicopathological parameters in colorectal cancer patients

\begin{tabular}{|c|c|c|c|c|c|}
\hline \multirow[t]{2}{*}{ Variables } & \multicolumn{5}{|c|}{ RAC1-GTP expression } \\
\hline & Total cases & Low & High & $\chi^{2}$ & $p$ value \\
\hline \multicolumn{6}{|l|}{ Gender } \\
\hline Female & 56 & 29 & 27 & 0.000 & 0.987 \\
\hline Male & 91 & 47 & 44 & & \\
\hline \multicolumn{6}{|l|}{ Age } \\
\hline$>58$ & 82 & 45 & 37 & 0.750 & 0.387 \\
\hline$\leq 58$ & 65 & 31 & 34 & & \\
\hline \multicolumn{6}{|c|}{ Recurrence status } \\
\hline With & 22 & 11 & 11 & 4.096 & 0.043 \\
\hline Without & 125 & 86 & 39 & & \\
\hline \multicolumn{6}{|c|}{ Histological grade } \\
\hline Well & 13 & 8 & 5 & 2.502 & 0.286 \\
\hline Moderate & 108 & 58 & 50 & & \\
\hline Poor & 25 & 10 & 16 & & \\
\hline \multicolumn{6}{|l|}{$\mathrm{T}$ stage } \\
\hline $\mathrm{T} 1$ & 4 & 2 & 2 & 16.888 & 0.001 \\
\hline $\mathrm{T} 2$ & 56 & 39 & 17 & & \\
\hline $\mathrm{T} 3$ & 85 & 32 & 53 & & \\
\hline $\mathrm{T} 4$ & 2 & 0 & 2 & & \\
\hline \multicolumn{6}{|l|}{$\mathrm{N}$ stage } \\
\hline No & 117 & 68 & 49 & 9.460 & 0.002 \\
\hline N1-2 & 30 & 8 & 22 & & \\
\hline \multicolumn{6}{|l|}{ M stage } \\
\hline M0 & 110 & 72 & 38 & 33.107 & 0.000 \\
\hline M1 & 37 & 4 & 33 & & \\
\hline \multicolumn{6}{|l|}{ TNM } \\
\hline I & 47 & 36 & 11 & 38.657 & 0.000 \\
\hline II & 49 & 30 & 19 & & \\
\hline III & 14 & 6 & 8 & & \\
\hline IV & 37 & 4 & 33 & & \\
\hline
\end{tabular}

( $p<0.001$ for all); and the invaded cells were $86 \pm 20$ vs. $171 \pm 12$ for HCT116, $84 \pm 49$ vs. $180 \pm 25$ for SW480, and $104 \pm 10$ vs. $156 \pm 14$ for SW620 $(p<0.001$ for all; Fig. 2b-e and S2b, c). These results suggest that RAC1GTP may be important in CRC invasion and metastasis.

\section{RAC1-GTP promotes EMT of CRC cells}

It has been demonstrated that the EMT process is crucial for CRC migration and invasion [22, 23]. We therefore analyzed the effect of RAC1-GTP disruption on the expression of EMT-related genes. The results show that NSC23766 treatment resulted in the upregulation of E-cadherin, a marker of epithelial phenotype, and downregulation of Vimentin and N-cadherin, two markers of mesenchymal phenotype in HCT116 and SW480 cells (Fig. 3a-c and S3). The expression of EMT-related transcription factors, including Slug and Snail, was also significantly decreased in NSC23766-treated CRC cells (Fig. 3a-c and S3). Since matrix metalloproteinases (MMPs), especially MMP9, have been reported to be involved in the invasion and metastasis of CRC [24], we determined the effect of RAC1-GTP on MMP9 expression. The results show that the inhibition of RAC1-GTP by NSC23766 significantly decreased MMP9 expression at both mRNA and protein level (Fig. 3a-c and S3). These results suggest that RAC1-GTP may regulate EMT in CRC cells to affect CRC invasion and metastasis.

\section{RAC1-GTP interacts with STAT3 to promote EMT of CRC cells}

It is well known that the STAT3 pathway plays an important role in regulating the EMT process in many types of cancers [25]. RAC1-GTP can directly activate the STAT3 pathway by binding to STAT3 or indirectly activate STAT3 by inducing interleukin (IL)-6 expression [13, 14, 26, 27]. We thus investigated whether RAC1-GTP could promote
Table 2 Univariate and multivariate analyses of the contribution of RAC1-GTP to the survival of colorectal cancer patients

\begin{tabular}{|c|c|c|c|c|c|c|c|c|}
\hline \multirow[t]{3}{*}{ Prognostic variables } & \multicolumn{4}{|c|}{ Univariate analysis } & \multicolumn{4}{|c|}{ Multivariate analysis } \\
\hline & \multirow[t]{2}{*}{$p$ value } & \multirow[t]{2}{*}{ HR } & \multicolumn{2}{|c|}{ 95\% CI for $\mathrm{HR}$} & \multirow[t]{2}{*}{$p$ value } & \multirow[t]{2}{*}{ HR } & \multicolumn{2}{|c|}{$95 \% \mathrm{CI}$ for $\mathrm{HR}$} \\
\hline & & & Lower & Upper & & & Lower & Upper \\
\hline Age & 0.013 & 2.197 & 1.177 & 4.103 & 0.033 & 2.003 & 1.057 & 3.794 \\
\hline RAC1-GTP & 0.000 & 5.244 & 2.866 & 9.596 & 0.000 & 3.289 & 1.707 & 6.338 \\
\hline Recurrence & 0.000 & 5.008 & 2.780 & 9.023 & 0.002 & 2.747 & 1.469 & 5.140 \\
\hline Tumor region & 0.001 & 3.032 & 1.607 & 5.718 & 0.022 & 2.154 & 1.119 & 4.145 \\
\hline Lymph node & 0.003 & 2.198 & 1.313 & 3.681 & 0.877 & 1.050 & 0.563 & 1.958 \\
\hline Location & 0.531 & 1.200 & 0.678 & 2.123 & 0.671 & 1.144 & 0.615 & 2.131 \\
\hline Gender & 0.352 & 0.752 & 0.412 & 1.370 & 0.181 & 0.651 & 0.347 & 1.220 \\
\hline Histological grade & 0.020 & 1.936 & 1.112 & 3.369 & 0.309 & 1.361 & 0.751 & 2.466 \\
\hline
\end{tabular}

$H R$ hazards ratio, $C I$ confidence interval 
Fig. 2 Inhibition of RAC1-GTP suppresses the migration and invasion of CRC cells in vitro. a Western blot analysis shows decreased RAC1-GTP expression by treatment with NSC23766, a specific inhibitor for RAC1-GTP, in CRC cells. b, c Migration analysis of HCT116 and SW480 CRC cells treated with or without NSC23766.

Scale bar $=100 \mu \mathrm{m}$. Data are presented as the mean \pm SD from three independent experiments. d, e Invasion assay of HCT116 and SW480 CRC cells treated with or without NSC23766. Scale bar $=100 \mu \mathrm{m}$. Data are presented as the mean \pm SD from three independent experiments. $* * * p<0.001$ a

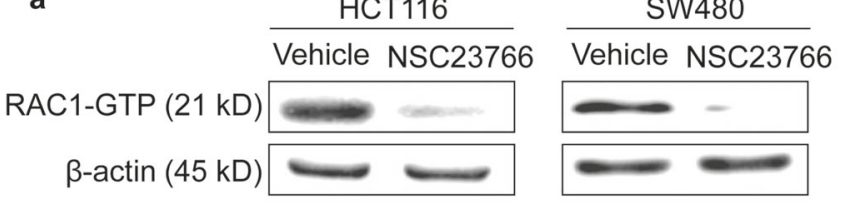

b
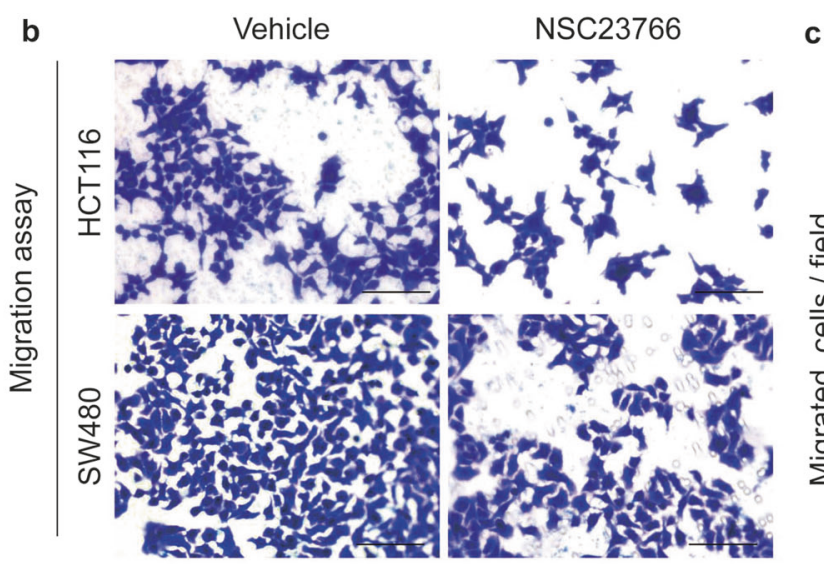

c
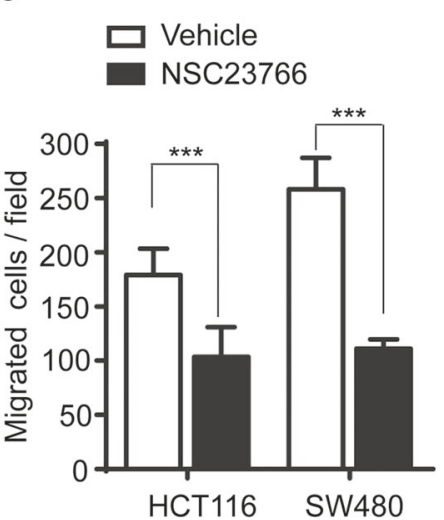

d

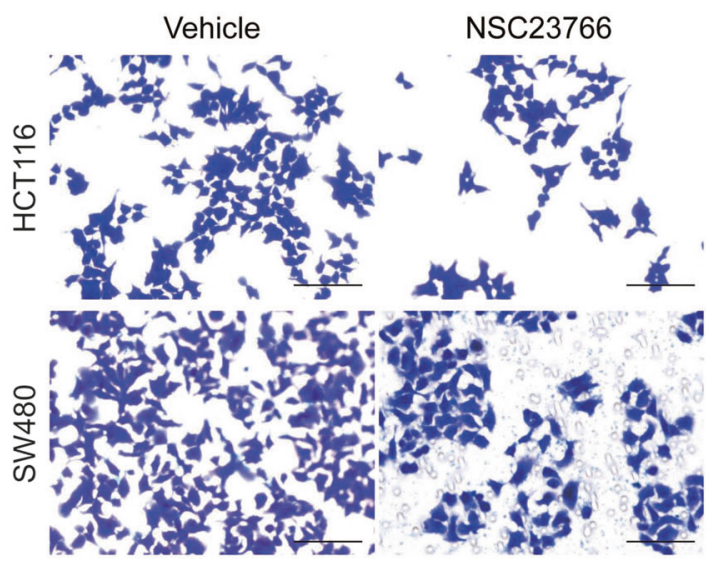

e

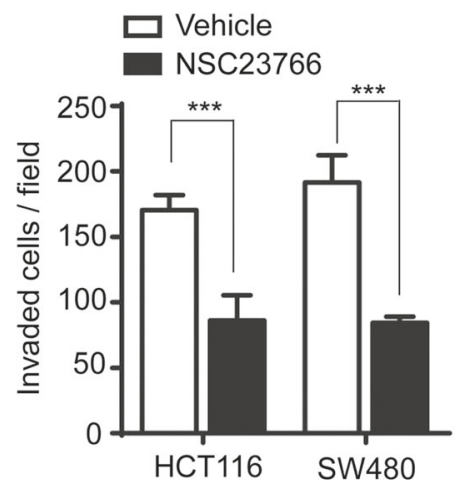

EMT by regulating STAT3 in CRC cells. The results show that NSC23766 treatment markedly suppressed the phosphorylation of STAT3 (p-STAT3-Tyr705) in HCT116, SW480, and SW620 cells (Fig. 4a and S3). Co-IP analyses indicated that RAC1-GTP directly bound to STAT3 in HCT116 cells (Fig. 4b), implying that RAC1-GTP promoted the EMT process by directly activating STAT3. Treatment with BP-1-102, a specific small molecular inhibitor of STAT3 phosphorylation [28], significantly attenuated migration and invasion (Fig. 4c, d and S4a, b) and reversed the EMT phenotype in HCT116 and SW480 cells (Fig. 4e). Similar results were observed in SW620 cells (Figure S5a-c). Overexpression of STAT3-C (Figure S6a) significantly rescued the suppressive effect of NSC23766 on the migration and invasion of HCT116 and SW480 cells (Fig. 4f, g and S6b, c). STAT3-C overexpression also reversed the EMT phenotype and MMP9 expression inhibited by NSC23766 (Fig. 4h). Similar results were observed in SW620 cells (Figure S7a-e). These results indicate that RAC1-GTP promotes EMT in CRC cells through activation of the STAT3 pathway.

\section{NSC23766 decreases invasion and the EMT phenotype of CRC xenograft in vivo}

We further evaluated the role of RAC1-GTP on CRC invasion in vivo by using a mouse subcutaneous xenograft model of HCT116. As shown in Fig. 5a, the xenograft tumors derived from animals treated with NSC23766 exhibited a clear boundary between tumor tissues and adjacent mouse tissues, while significant infiltrations of tumor cells into the adjacent muscle tissues were observed in the animal treated with vehicle (Fig. 5a). Western blot analyses and immunohistochemistry confirmed that RAC1GTP expression was significantly reduced in the tumors from the mice treated with NSC23766 compared to those treated with vehicle (Fig. 5b, c). Importantly, NSC23766 treatment enhanced the expression of the epithelial marker 
Fig. 3 Inhibition of RAC1-GTP suppresses the EMT process of CRC cells. a, b qRT-PCR analysis shows that NSC23766 treatment resulted in upregulated E-cadherin (E-cad) and downregulated N-cadherin (Ncad), Vimentin, Slug, Snail, and MMP9 compared to vehicle treatment in HCT116 and SW480 CRC cells. c Western blot analysis revealed the upregulation of E-cadherin and downregulation of N-cadherin, Vimentin, Snail, Slug, MMP9, and RAC1-GTP in NSC23766treated cells. $* p<0.05 ; * * p<$ $0.01 ; * * * p<0.001$
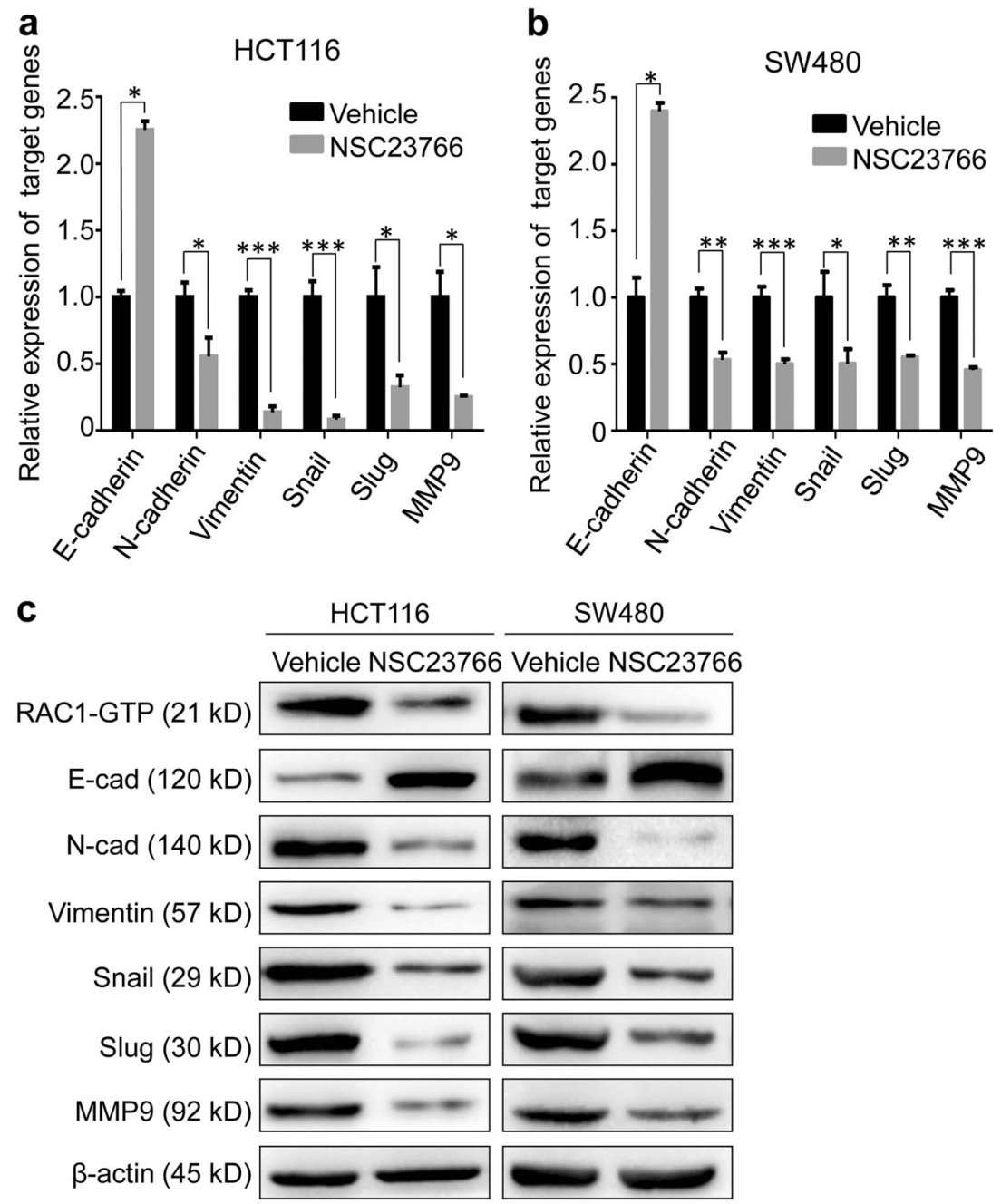

E-cadherin, but decreased the expression of mesenchymal markers N-cadherin as well as EMT-related transcription factor Slug in the CRC xenografts (Fig. 5b, c). The level of p-STAT3 was also decreased in the xenografts treated with NSC23766 as compared with the vehicle control (Fig. 5b, c). These results confirm the role of RAC1-GTP in promotion of EMT and invasion in CRC. Given that EMTmediated tumor invasion significantly contributes to tumor growth and malignant progression [29, 30], we further determined the effect of RAC1-GTP disruption on CRC tumor growth. Disrupting RAC1-GTP by NSC23766 treatment significantly inhibited tumor growth, as demonstrated by the reduced tumor volume and tumor weight in mice treated with NSC23766 relative to those treated with vehicle control (Figure S8a, b, and c). Consistently, NSC23766 treatment also significantly suppressed the proliferation of HCT116 CRC cells in vitro (Figure S8d). Collectively, our results indicate that RAC1-GTP is a potential therapeutic target to inhibit the EMT-mediated invasion and tumor growth of CRC.

\section{Discussion}

We previously demonstrated that the active form of RAC1 (RAC1-GTP) is involved in maintenance of stemness in CRC stem cells [18]. In this study, we found that RAC1GTP was highly expressed in CRC cancerous tissues and its expression level was significantly correlated with TNM stages, lymph node spread, and distant metastasis as well as patient survival. To our knowledge, this is the first clinicopathological study to link RAC1-GTP to the clinicopathological features of CRC and the outcome of CRC patients. RAC1-GTP is the active form of RAC1 [12]. It has been reported that $\mathrm{RAC1}$ expression is important for the invasion and metastasis of CRC [31, 32], but in our study, no significant difference of the expression of RAC1 at mRNA level between cancerous and adjacent normal tissues was observed. Hence, our results suggest that RAC1-GTP may be the main form of RAC1 involved in CRC progression.

Increasing evidence suggests that the EMT process is associated with gaining the capability of invasion and 
a

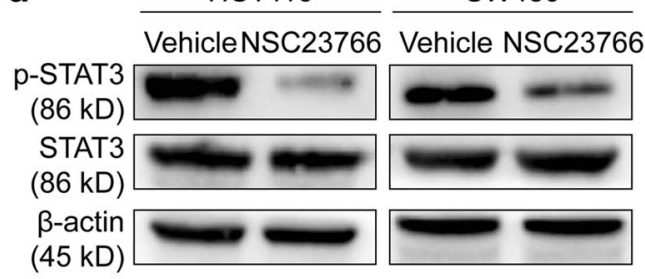

b

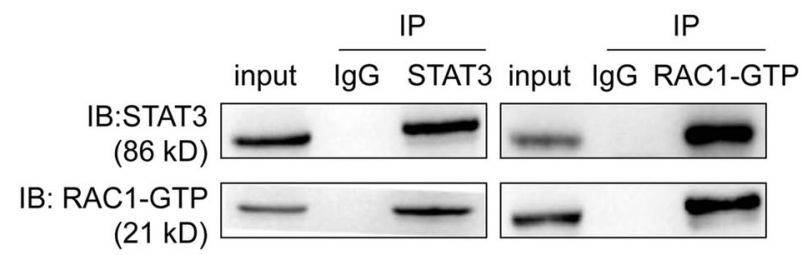

e

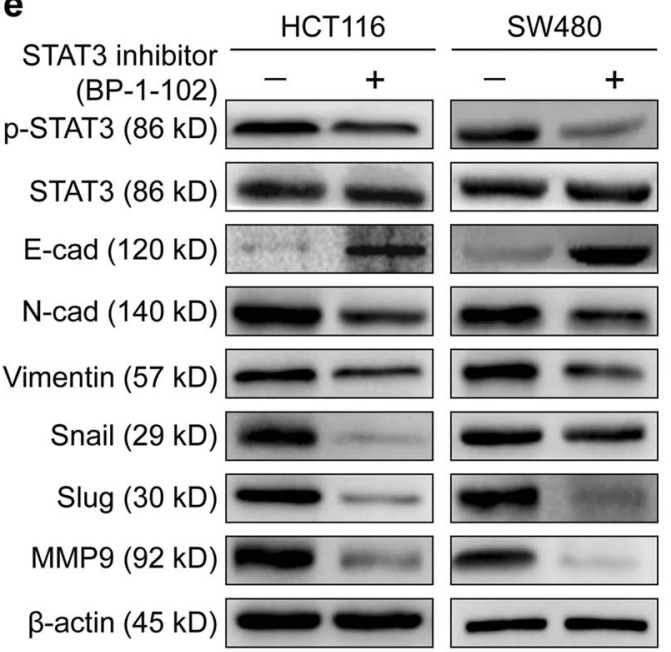

h

g

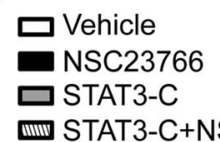

Vehicle

STAT3 inhibitor (BP-1-102)
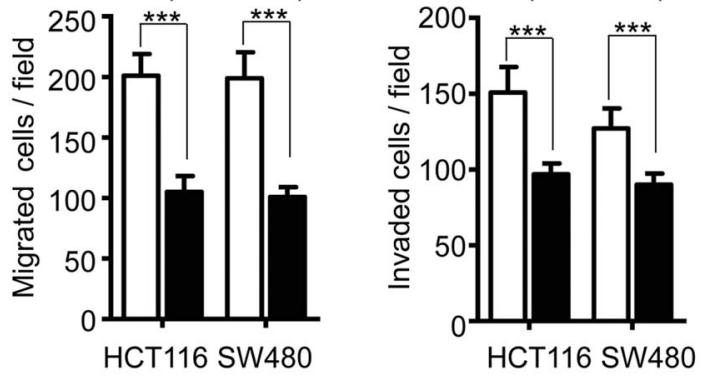

f

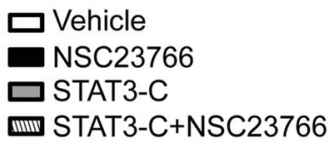

InI STAT3-C+NSC23766
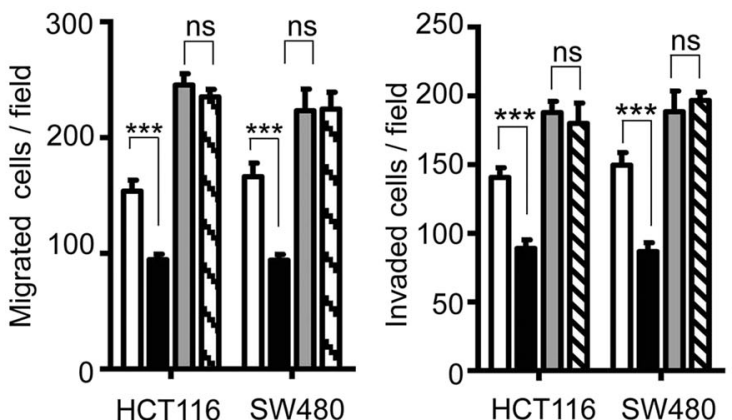

Fig. 4 RAC1-GTP promotes EMT of CRC cells by directly binding to STAT3. a Western blot analysis revealed that NSC23766 treatment markedly decreased STAT3 phosphorylation (p-STAT3-Tyr705) in HCT116 and SW480 cells. b Co-immunoprecipitation assay shows the interaction between RAC1-GTP and STAT3. c Quantification of migration analysis of HCT116 and SW480 cells treated with or without BP-1-102. The data are presented as the mean \pm SD from three independent experiments. d Quantification of invasion analysis of HCT116 and SW480 cells treated with or without BP-1-102. The data are presented as the mean \pm SD from three independent experiments. $\mathbf{e}$ Western blot analysis revealed upregulation of E-cadherin and downregulation of p-STAT3, N-cadherin (N-cad), Vimentin, Snail,

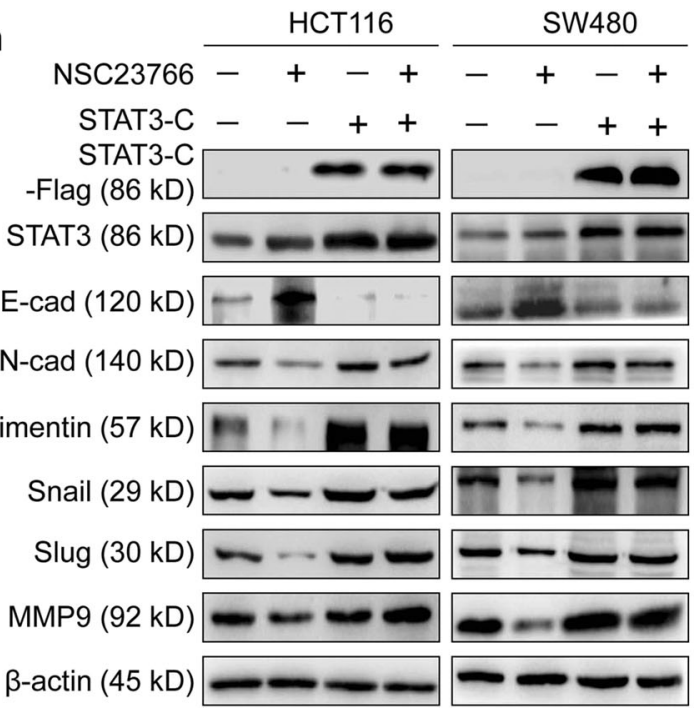

Slug as well as MMP9 treated by BP-1-102 in HCT116 and SW480 cells. f Migration analysis of HCT116 and SW480 cells transfected with or without STAT3-C and treated with or without NSC23766. Data are presented as the mean \pm SD from three independent experiments. g Invasion analysis of HCT116 and SW480 cells transfected with or without STAT3-C and treated with or without NSC23766. Data are presented as the mean \pm SD from three independent experiments. h Western blot shows that ectopic expression of STAT3-C rescued the EMT-related molecules and MMP9 altered by NSC23766 treatment in HCT116 and SW480 cells. $* * * p<0.001$; ns no significance 

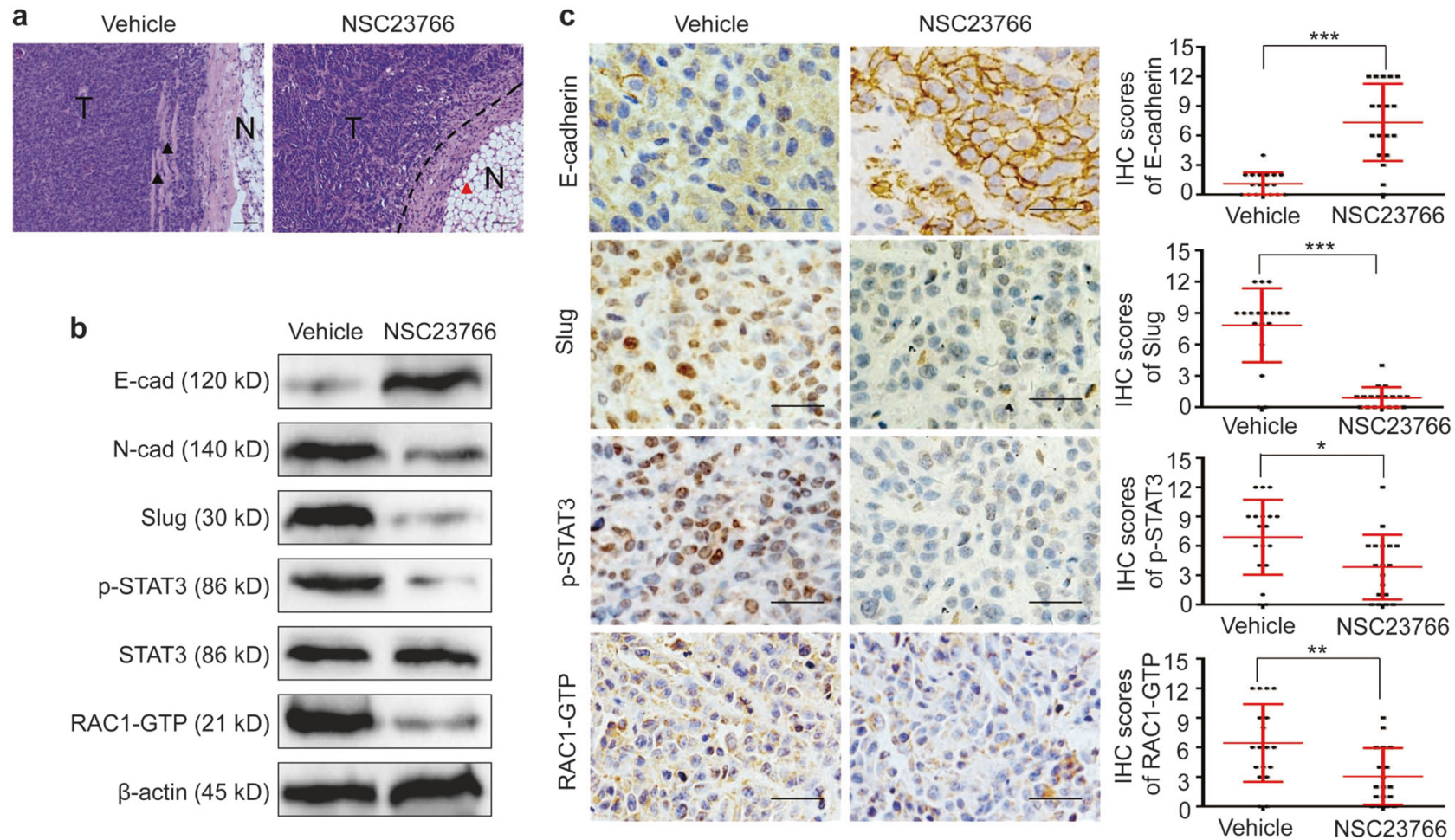

Fig. 5 Targeting RAC1-GTP by NSC23766 suppresses the EMTmediated invasion of HCT116 CRC cells in vivo. a Representative images of H\&E staining in xenograft specimens. Tumors of vehicletreatment group invaded into adjacent muscle tissue, while tumors of NSC23766-treatment group showed clear boundary between tumor tissues and adjacent normal tissues ( $\mathrm{T}$ indicates tumor tissues; $\mathrm{N}$ indicates adjacent normal tissues). Muscle tissue was indicated by black triangle, and peripheral adipose tissue was indicated by red

metastasis in CRC cells [33]. The major molecular features of EMT are the downregulation of the epithelial marker Ecadherin and the upregulation of mesenchymal markers, such as Vimentin and N-cadherin, as well as EMT-related transcription factors, such as Snail and Slug [34]. We found that disrupting RAC1-GTP increased the expression of epithelial marker E-cadherin but decreased the levels of mesenchymal markers, which was consistent with previous studies showing that RAC1-GTP regulated E-cadherinmediated adherent junctions in pancreatic carcinoma cells [35]. In addition, the maintenance of epithelial phenotype of cells relies on close contacts with extracellular matrix [36], and MMPs can degrade extracellular matrix proteins thus promote tumor invasion and metastasis [35, 37]. Our results demonstrated that RAC1-GTP disruption also significantly reduced the expression of MMP9, an important member of MMPs in CRC [24].

STAT3 is proposed to be involved in the EMT process. Inhibition of STAT3 phosphorylation significantly increases E-cadherin expression but decreases $\mathrm{N}$-cadherin and Vimentin expression in highly invasive LoVo CRC cells [38, 39]. Our results show that inhibition of RAC1-GTP by NSC23766 suppressed STAT3 phosphorylation in three triangle. Scale bar $=100 \mu \mathrm{m}$. b Western blot showing that NSC23766 upregulated the expression of E-cadherin (E-cad), but downregulated the expressions of $\mathrm{N}$-cadherin (N-cad), Slug, p-STAT3, STAT3, and RAC1-GTP in CRC xenografts. $\mathbf{c}$ Representative IHC staining images (left panels) and quantification (right panels) of E-cadherin, Slug, pSTAT3, and RAC1-GTP expressions in CRC xenografts. Scale bar = $50 \mu \mathrm{m} . * p<0.05 ; * * p<0.01 ; * * * p<0.001$

CRC cell lines, and introduction of STAT3-C significantly attenuated the suppressive effect of RAC1-GTP disruption on the EMT-mediated migration and invasion of CRC cells, underscoring the role of RAC1-GTP-STAT3 pathway in the regulation of EMT process. Previous reports suggested that there were two possible mechanisms underlying the regulation of RAC1-GTP on STAT3 activity: (1) active RAC1 promotes IL-6 expression and autocrine IL-6 further activates the STAT3 pathway [13]; and (2) RAC1-GTP directly binds to STAT3 to regulate STAT3 phophorylation $[14,26$, 27]. Our results demonstrate that RAC1-GTP directly interacted with STAT3 to promote its phosphorylation, while disrupting RAC1-GTP activity by NSC23766 had a minimal effect on IL-6 expression in CRC cells (data not shown).

In conclusion, RAC1-GTP is an important regulator in invasion of CRC by directly activating STAT3 pathway to induce EMT. Although our results suggest that RAC1-GTP may serve as a new prognostic indicator and a potential therapeutic target for CRC, its clinical relevance needs to be investigated in independent prospective clinical studies.

Acknowledgements This research was supported by the grant from China National Natural Science Foundation of China (No. 81402460). 


\section{Compliance with ethical standards}

Conflict of interest The authors declare that they have no conflict of interest.

\section{References}

1. Ferlay J, Shin HR, Bray F, et al. Estimates of worldwide burden of cancer in 2008. Int J Cancer. 2010;127:2893-17.

2. Stein U, Schlag PM. Clinical, biological, and molecular aspects of metastasis in colorectal cancer. Recent Results Cancer Res. 2007;176:61-80.

3. Siegel R, DeSantis C, Virgo K, et al. Cancer treatment and survivorship statistics, 2012. CA Cancer J Clin. 2012;62:220-41.

4. Cao H, Xu E, Liu H, et al. Epithelial-mesenchymal transition in colorectal cancer metastasis: a system review. Pathol Res Pract. 2015;211:557-69.

5. Han X, Wang L, Ning Y, et al. Long non-coding RNA AFAP1AS1 facilitates tumor growth and promotes metastasis in colorectal cancer. Biol Res. 2016;49:36.

6. Colomiere M, Ward AC, Riley C, et al. Cross talk of signals between EGFR and IL-6R through JAK2/STAT3 mediate epithelial-mesenchymal transition in ovarian carcinomas. $\mathrm{Br} \mathbf{J}$ Cancer. 2009;100:134-44.

7. Qi J, Yu Y, Akilli Öztürk Ö, et al. New Wnt/beta-catenin target genes promote experimental metastasis and migration of colorectal cancer cells through different signals. Gut. 2016;65:1690-701.

8. Liu XL, Zhang XT, Meng J, et al. ING5 knockdown enhances migration and invasion of lung cancer cells by inducing EMT via EGFR/PI3K/Akt and IL-6/STAT3 signaling pathways. Oncotarget. 2017;8:54265-76.

9. Chae YC, Kim JH, Kim KL, et al. Phospholipase D activity regulates integrin-mediated cell spreading and migration by inducing GTP-Rac translocation to the plasma membrane. Mol Biol Cell. 2008;19:3111-23.

10. Collins C, Tzima E. Rac[e] to the pole: setting up polarity in endothelial cells. Small GTPases. 2014;5:e28650.

11. Nobes CD, Hall A. Rho, rac, and cdc42 GTPases regulate the assembly of multimolecular focal complexes associated with actin stress fibers, lamellipodia, and filopodia. Cell. 1995;81:53-62.

12. Bosco EE, Mulloy JC, Zheng Y. Rac1 GTPase: a "Rac" of all trades. Cell Mol Life Sci. 2009;66:370-4.

13. Faruqi TR, Gomez D, Bustelo XR, et al. Rac1 mediates STAT3 activation by autocrine IL-6. Proc Natl Acad Sci USA. 2001;98:9014-9.

14. Simon AR, Vikis HG, Stewart S, et al. Regulation of STAT3 by direct binding to the Rac1 GTPase. Science. 2000;290:144-7.

15. Han Q, Leng J, Bian D, et al. Rac1-MKK3-p38-MAPKAPK2 pathway promotes urokinase plasminogen activator mRNA stability in invasive breast cancer cells. J Biol Chem. 2002;277:48379-85.

16. Shi Y, Zhou W, Cheng L, et al. Tetraspanin CD9 stabilizes gp130 by preventing its ubiquitin-dependent lysosomal degradation to promote STAT3 activation in glioma stem cells. Cell Death Differ. 2017;24:167-80.

17. Shi Y, Chen C, Zhang X, et al. Primate-specific miR-663 functions as a tumor suppressor by targeting PIK3CD and predicts the prognosis of human glioblastoma. Clin Cancer Res. 2014;20:1803-13.

18. Rao J, Zhou ZH, Yang J, et al. Semaphorin-3F suppresses the stemness of colorectal cancer cells by inactivating Rac1. Cancer Lett. 2015;358:76-84.

19. Veluthakal R, Sidarala V, Kowluru A. NSC23766, a known inhibitor of Tiam1-Rac1 signaling module, prevents the onset of
Type 1 diabetes in the NOD mouse model. Cell Physiol Biochem. 2016;39:760-7.

20. Wu F, Zhou Q, Yang J, et al. Endogenous axon guiding chemorepulsant semaphorin-3F inhibits the growth and metastasis of colorectal carcinoma. Clin Cancer Res. 2011;17:2702-11.

21. Igarashi T, Araki K, Yokobori T, et al. Association of RAB5 overexpression in pancreatic cancer with cancer progression and poor prognosis via E-cadherin suppression. Oncotarget. 2017;8:12290-300.

22. Wang $\mathrm{L}, \mathrm{Yu}$ P. miR-300 promotes proliferation and EMTmediated colorectal cancer migration and invasion by targeting p53. Oncol Rep. 2016;36:3225-32.

23. Li J, Zou K, Yu L, et al. MicroRNA-140 inhibits the epithelialmesenchymal transition and metastasis in colorectal cancer. Mol Ther Nucleic Acids. 2018;10:426-37.

24. Chu D, Zhao Z, Zhou Y, et al. Matrix metalloproteinase-9 is associated with relapse and prognosis of patients with colorectal cancer. Ann Surg Oncol. 2012;19:318-25.

25. Yuan J, Zhang F, Niu R. Multiple regulation pathways and pivotal biological functions of STAT3 in cancer. Sci Rep. 2015;5:17663.

26. Zhao L, Du X, Huang K, et al. Rac1 modulates the formation of primordial follicles by facilitating STAT3-directed Jagged1, GDF9 and BMP15 transcription in mice. Sci Rep. 2016;6:23972.

27. Mattagajasingh SN, Yang XP, Irani K, et al. Activation of Stat 3 in endothelial cells following hypoxia-reoxygenation is mediated by Rac1 and protein kinase C. Biochim Biophys Acta. 2012;1823:997-1006.

28. Zhang X, Yue P, Page BD, et al. Orally bioavailable smallmolecule inhibitor of transcription factor Stat3 regresses human breast and lung cancer xenografts. Proc Natl Acad Sci USA. 2012;109:9623-8.

29. Caramel J, Papadogeorgakis E, Hill L, et al. A switch in the expression of embryonic EMT-inducers drives the development of malignant melanoma. Cancer Cell. 2013;24:466-80.

30. Gujral TS, Chan M, Peshkin L, et al. A noncanonical Frizzled2 pathway regulates epithelial-mesenchymal transition and metastasis. Cell. 2014;159:844-56.

31. Espina C, Céspedes MV, García-Cabezas MA, et al. A critical role for Rac1 in tumor progression of human colorectal adenocarcinoma cells. Am J Pathol. 2008;172:156-66.

32. Bao Y, Guo H, Lu Y, et al. Blocking hepatic metastases of colon cancer cells using an shRNA against Rac1 delivered by activatable cell-penetrating peptide. Oncotarget. 2016;7:77183-95.

33. Gurzu S, Silveanu C, Fetyko A, et al. Systematic review of the old and new concepts in the epithelial-mesenchymal transition of colorectal cancer. World J Gastroenterol. 2016;22:6764-75.

34. Vu T, Datta PK. Regulation of EMT in colorectal cancer: a culprit in metastasis. Cancers (Basel). 2017;9:E171.

35. Hage B, Meinel K, Baum I, et al. Rac1 activation inhibits Ecadherin-mediated adherens junctions via binding to IQGAP1 in pancreatic carcinoma cells. Cell Commun Signal. 2009;7:23.

36. Schmitz AA, Govek EE, Bottner B, et al. Rho GTPases: signaling, migration, and invasion. Exp Cell Res. 2000;26:1-12.

37. Chabottaux V, Noel A. Breast cancer progression: insights into multifaceted matrix metalloproteinases. Clin Exp Metastas-. 2007;24:647-56.

38. Xiong H, Hong J, Du W, et al. Roles of STAT3 and ZEB1 proteins in E-cadherin down-regulation and human colorectal cancer epithelial-mesenchymal transition. J Biol Chem. 2012;287:5819-32.

39. Xiong $\mathrm{H}$, Zhang $\mathrm{ZG}$, Tian $\mathrm{XQ}$, et al. Inhibition of JAK1, 2/STAT3 signaling induces apoptosis, cell cycle arrest, and reduces tumor cell invasion in colorectal cancer cells. Neoplasia. 2008;10:287-97. 